\title{
Oncological safety of platelet-rich fibrin application on the pharyngeal closure after total laryngectomy
}

\author{
Ahmed Mostafa Eid ${ }^{1} \cdot$ Hisham Atef Ebada ${ }^{1}$ (D) $\cdot$ Ahmed Musaad Abd El-Fattah ${ }^{1} \cdot$ Ali Tawfik $^{1}$
}

Received: 1 December 2020 / Accepted: 11 December 2020 / Published online: 2 January 2021

(c) The Author(s), under exclusive licence to Springer-Verlag GmbH, DE part of Springer Nature 2021

To the editor,

We thank Spartalis and Troupis [1], for their constructive comments on our article "Platelet-rich fibrin: an autologous biomaterial for healing assistance of pharyngeal repair in total laryngectomy" [2] and the editor for giving us the opportunity to reply.

Spartalis and Troupis [1], believe that the release of growth factors from the platelet-rich fibrin (PRF), stimulates angiogenesis, induces tumor lymphangiogenesis, enhances tumorigenesis, proliferation, and survival. However, we believe that this would be true if PRF is directly applied to the same tissue from which the tumor has developed. An example to this is the concern which have been raised regarding the extent of cellular promotion and the corresponding potential malignant transformation when platelet-rich plasma (PRP) was applied on the breast tissue after lumpectomy [3]. In laryngeal carcinoma, the larynx is removed in toto, then the PRF is not applied on any laryngeal tissue. It is applied to the pharyngeal repair to enhance healing. Furthermore, frozen section biopsies are routinely taken from the residual pharyngeal wall before repair to make sure that the margins are free.

Also, it is well known that chronic exposure to carcinogens leads to the development of several primary tumors [4]. The life span of platelets is $8-10$ days. Consequently, this short life span allows for enhancing tissue healing in first few days postoperatively, without the risk of the chronic longterm effect on the tissue that would enhance tumorigenesis.

Lastly, although many articles have studied the potential role of different growth factors in the development of laryngeal carcinoma, the exact pathogenesis of laryngeal

This reply refers to the comment available online at https://doi. org/10.1007/s00405-020-06469-8.

Hisham Atef Ebada

hishamebada@mans.edu.eg

1 Otorhinolaryngology Department, Faculty of Medicine,

Mansoura University, Mansoura 35511, Egypt carcinoma is still not fully understood [5]. Further studies are needed to explore the potential risk of growth factors derived from PRF in enhancing tumorigenesis.

Funding No funding was received for this work.

\section{Compliance with ethical standards}

Conflict of interest The authors declare that they have no conflict of interest.

\section{References}

1. Spartalis E, Troupis T (2020) Anatomical considerations regarding the oncological safety of platelet-rich fibrin application at the tumor bed after total laryngectomy for advanced carcinoma. Eur Arch Otorhinolaryngol, p. 1-2

2. Eid AM, Ebada HA, Abd El-Fattah AM, Tawfik A (2020) Plateletrich fibrin: an autologous biomaterial for healing assistance of pharyngeal repair in total laryngectomy. Eur Arch Otorhinolaryngol, p. 1-8

3. Spartalis E, Tsilimigras DI, Charalampoudis P, Karachaliou G-S, Moris D, Athanasiou A, Spartalis M, Bolkas V, Dimitroulis D, Nikiteas N (2017) The "Yin and Yang" of platelet-rich plasma in breast reconstruction after mastectomy or lumpectomy for breast cancer. Anticancer Res 37(12):6557-6562

4. Fujiwara K, Yazama H, Donishi R, Koyama S, Fukuhara T, Takeuchi H (2020) Inhibitory effects of glucosylceramide on tumorigenesis induced by a carcinogen in mice. Laryngoscope 130(11):E593-E597

5. Ma K, Sun X, Ma L, Zhang S (2020) Expression of serum PTTG1 in laryngeal carcinoma and its correlation to prognosis. Clin Exp Otorhinolaryngol 13(1):64

Publisher's Note Springer Nature remains neutral with regard to jurisdictional claims in published maps and institutional affiliations. 